\title{
Anabases
}

\section{La guérison dans les sanctuaires du monde grec antique : de Meibom aux Edelstein, remarques historiographiques}

Pierre Sineux

\section{CpenEdition}

Journals

Édition électronique

URL : http://journals.openedition.org/anabases/1713

DOI : 10.4000/anabases. 1713

ISSN : 2256-9421

Éditeur

E.R.A.S.M.E.

\section{Édition imprimée}

Date de publication : 1 mars 2011

Pagination : 11-25

ISSN : $1774-4296$

\section{Référence électronique}

Pierre Sineux, «La guérison dans les sanctuaires du monde grec antique : de Meibom aux Edelstein, remarques historiographiques », Anabases [En ligne], 13 | 2011, mis en ligne le 01 mars 2014, consulté le 20 octobre 2019. URL : http://journals.openedition.org/anabases/1713; DOI : 10.4000/anabases. 1713

Ce document a été généré automatiquement le 20 octobre 2019

(c) Anabases 


\title{
La guérison dans les sanctuaires du monde grec antique : de Meibom aux Edelstein, remarques historiographiques
}

\author{
Pierre Sineux
}

1 Jusqu'à la fin du XIX ${ }^{\mathrm{e}}$ siècle, l'activité des sanctuaires guérisseurs de la Grèce antique était analysée, pour l'essentiel, en termes d'histoire de la pratique médicale. Et ce d'autant que, depuis la fin du XVIII ${ }^{\mathrm{e}}$ siècle, ce sont les médecins, plus ou moins nourris d'« humanités ", qui occupent largement le terrain de la production « historique » sur les sanctuaires guérisseurs; ils voient dans les guérisons des sanctuaires des éléments de l'histoire (ou de la préhistoire) de leur discipline. Dans cette optique, les études de ces sanctuaires guérisseurs sont alors principalement centrées sur les formes que prend l'intervention humaine dans la guérison et sur la relation qui s'établit entre les prêtres, les médecins et les malades.

2 La publication dans les années 1880 des "stèles de guérison" d'Épidaure pouvait conduire à une rupture; dans sa "Chronique d'Orient» parue dans la Revue Archéologique en 1884, c'est en ces termes que Salomon Reinach rendait compte de leur découverte à Épidaure par P. Cavvadias : "C'est dans le voisinage de ce monument [le portique dit d'incubation] que l'heureux explorateur a trouvé les deux grandes stèles avec inscriptions indiquant les noms des malades traités dans l'Asclépieion et les merveilleuses guérisons opérées par le dieu... L'inscription contient le récit de vingt guérisons ou plutôt de vingt miracles, car suivant la juste remarque de l'éditeur, il n'est nulle part question de remèdes pharmaceutiques, mais seulement de visions et de songes ${ }^{1}$.»

3 En feignant d'hésiter entre les termes « miracle » et " guérison » pour qualifier les faits consignés sur les stèles découvertes à Épidaure, Salomon Reinach semblait, à la suite de P. Cavvadias, donner le signal du tournant que l'étude de l'activité des sanctuaires asclépieiens pouvait prendre: le champ de l'histoire de la médecine semblait devoir 
être abandonné, ce qui supposait l'ouverture vers d'autres champs disciplinaires dans lequel l'histoire des religions semblait devoir s'imposer. C'est ce tournant qu'il convient d'interroger en suivant le fil des principales parutions sur la question de la guérison dans les sanctuaires du monde grec jusqu'à la publication, en 1945, de la somme de E.J. et L. Edelstein ${ }^{2}$ qui a, longtemps, servi d'ouvrage de référence sur l'histoire du culte d'Asklépios dans le monde grec.

4 Faire entrer l'activité des sanctuaires guérisseurs dans le champ de l'histoire de la médecine était d'abord une manière d'éloigner le «fantôme du diable » qui planait sur l'Exercitatio de incubatione in fanis deorum medicinae causa olim facta du jeune Heinrich Meibom $^{3}$. Dans cette dissertation publiée en 1659, H. Meibom, en effet, ne voyait dans les guérisons que l'effet de l'action du diable, habitant selon lui les "démons païens ", et il rejetait toute intervention d'une forme de médecine rationnelle; il établissait alors une continuité entre les dieux " païens » et les saints catholiques dont l'action, écrivaitil, ne faisait que prolonger la supercherie des démons païens. C'est dans la deuxième moitié du XvIII ${ }^{\mathrm{e}}$ siècle, alors que se constituait le champ disciplinaire des sciences naturelles, que l'on a vu s'affirmer la conviction que les hommes seuls intervenaient dans le traitement des maladies. L'approche de la question des guérisons dans les sanctuaires de l'Antiquité grecque prenait alors une nouvelle orientation: il s'agissait désormais de trouver une cause aux guérisons en se fondant sur l'observation de la procédure suivie au cours du rite de l'incubation. Dans ce contexte, les théories de Franz Messmer sur le magnétisme animal et sur ses liens avec le somnambulisme allaient exercer une influence déterminante.

Dans son Mémoire sur la découverte du magnétisme animal publié en 1779, F. Messmer reprend une terminologie héritée de Paracelse qui lui permet de définir le «magnétisme animal » comme la capacité d'un individu, quel qu'il soit, à guérir son prochain grâce au fluide naturel qu'un magnétiseur, détenteur de techniques spécifiques, fait circuler dans un but thérapeutique. L'accumulation et la circulation du fluide se font grâce à des "passes ", dites "messmériennes "; ouvrant avec quelques adeptes une Société de l'Harmonie Uniververselle en 1782, F. Messmer se heurte à l'hostilité de la Faculté de Médecine et doit quitter la France en 1785. Ses idées néanmoins se diffusent quelques années avant la Révolution française puis sous le Premier Empire et la Restauration. C'est notamment à partir du magnétisme animal que le marquis de Puységur en vient à l'usage du «somnambulisme provoqué » ou « sommeil magnétique »-, où il s'appuie sur une expérience faite sur un jeune paysan qu'il avait endormi et dont il avait observé l'intense activité mentale ; le patient se met alors à diagnostiquer ses propres maux et ceux des autres malades et à prescrire les remèdes appropriés ${ }^{4}$.

6 Le succès de ces théories est tel que les guérisons dans les sanctuaires grecs ont pu être perçues comme autant de preuves de l'efficacité des méthodes thérapeutiques du somnambulisme et du sommeil "magnétique ». Les prêtres d'Asklépios sont alors considérés comme des sortes de précurseurs de Messmer ou du marquis de Puységur. C'est l'opinion défendue, par exemple, par une dissertatio de C.A. Koenig sur Aelius Aristide qui paraît en 1818: la maladie de celui-ci est qualifiée d'« ein hitziges Nervenfieber " pour la guérison de laquelle le somnambulisme apparaît donc tout à fait adapté5. En 1840, A. Gauthier, dans son Introduction au magnétisme, soutient que les prêtres des sanctuaires n'étaient que des magnétiseurs qui parvenaient à endormir les malades si bien que ceux-ci, dans leur sommeil, répondaient à leurs sollicitations 
verbales (prédiction de l'avenir, prescription de remèdes) et physiques (frictions, onctions, attouchements) ${ }^{6}$. Ces théories ménageaient ainsi une forme de rationalité au fondement de l'activité des sanctuaires guérisseurs et une relation particulière, de nature thérapeutique, entre les prêtres et les fidèles.

Toutefois, de même que les thérapies fondées sur le somnambulisme faisaient l'objet de vives critiques, le bien-fondé de leur application à l'incubation antique fut également rapidement contesté. Cette opposition sous-tend l'ouvrage que Louis-Philibert-Auguste Gauthier fait paraître en 1844 sous le titre Recherches historiques sur l'exercice de la médecine dans les temples, chez les peuples de l'Antiquité, suivies de considérations sur les rapports qui peuvent exister entre les guérisons qu'on obtenait dans les anciens temples, à l'aide des songes, et le magnétisme animal, et sur l'origine des hôpitaux ${ }^{7}$. Ce médecin de l'Antiquaille de Lyon (1792-1850) affirmait sa conviction que le somnambulisme magnétique ne figurait pas parmi les moyens utilisés par les prêtres des sanctuaires dans le traitement des maladies. Sa réfutation reposait sur le témoignage d'Aelius Aristide qui ne mentionne nulle part la présence de prêtres pendant le sommeil du malade. Il y oppose le rôle, essentiel à ses yeux, de la mémoire dans les guérisons des sanctuaires en contradiction totale avec l'oubli au réveil qui caractérise précisément l'expérience du somnambulisme.

8 Dans le cadre d'une réflexion plus générale sur l'origine de la médecine, les prêtres des sanctuaires étaient cependant crédités par L.-P.-A. Gauthier d'un rôle majeur : à une première étape de l'histoire de la médecine, purement "domestique et populaire ", aurait succédé la multiplication des "temples pour Esculape» dont les prêtres "ne tardèrent pas à devenir les seuls médecins ${ }^{8} »$. La question de la date du début du culte étant rapidement réglée (probablement pas antérieur à la guerre de Troie, peu de temps après sans doute...), l'essentiel de la thèse portait sur l'évolution de l'exercice de la médecine dans les sanctuaires: pendant une première période, antérieure à Hippocrate, les Asclépiades, qui se disaient descendants d'Esculape, auraient commencé " peu de temps après la fondation des temples... à y exercer la médecine, sous le voile de la superstition et du mystère "; puis, dans une seconde période, d'Hippocrate au christianisme, la médecine des temples se serait développée avant de dégénérer et de devenir une « jonglerie grossière ${ }^{9}$ ».

Les « prêtres-médecins » auraient ainsi joué selon L.-P.-A. Gauthier un rôle déterminant dans la guérison des malades. Ils agissaient d'abord sur leur imagination: "Il est probable que souvent les prêtres faisaient entendre dans le temple des paroles que des hommes crédules et à moitié endormis, dont l'imagination était fortement préoccupée, prenaient pour des songes ou pour des oracles ${ }^{10}$.» C'est moins par les remèdes employés que les guérisons pouvaient s'expliquer que par « les moyens propres à agir sur l'imagination de ceux qui venaient les consulter ». Parmi ces moyens, on range une partie des rites préliminaires au rite de l'incubation: jeûnes, purifications, bains, frictions... dont la pratique était considérée comme générale. Aux fêtes, organisées en l'honneur du dieu, « dont les pompes inspiraient le respect et exaltaient l'imagination ${ }^{11}$ ", était attribuée la fonction également de "donner plus de confiance à ceux qui venaient dans les temples ». Le primat donné à la force de l'auto-suggestion du malade lui-même n'empêchait pas qu'on reconnaissait une action plus directe des "prêtresmédecins »: la lecture littérale des passages d'Aristophane (Ploutos), d'Artémidore (Oneirocriticon) et des fragments d'Hippys de Rhégion ne fournissait-elle pas des preuves de ce que, "pendant que les malades dormaient ou feignaient de dormir, les prêtres 
leur faisaient des applications sur diverses parties du corps et même des opérations chirurgicales ${ }^{12} »$.

Toute l'attention de L.-P.-A. Gauthier pouvait alors se concentrer sur la question de la structuration du groupe des "prêtres d'Esculape » (ils auraient formé des collèges ou des corporations) et, surtout, sur la question de leur instruction, fondée d'abord sur une transmission familiale avant que des «étrangers » à "l'ordre » des Asclépiades ne soient admis à se faire initier: l'institution du Serment d'Hippocrate aurait alors marqué ce tournant à partir duquel les Asclépiades auraient commencé à admettre des étrangers dans leurs rangs. C'est à ces "prêtres » qu'on devrait la fondation, «dans leurs temples ", des écoles où ils auraient enseigné la médecine : celles de Cos, de Cnide, de Rhodes, et l'école italique. Leur formation s'appuyait sur la lecture des « inscriptions des temples ", l'habitude de voir un grand nombre de malades faisant le reste et «à la fin [devant] donner une certaine instruction médicale aux prêtres ${ }^{13}$ ».

11 Dans cette première moitié du $\mathrm{XIX}^{\mathrm{e}}$ siècle, l'analyse de l'activité des sanctuaires guérisseurs repose en fait sur la confusion entre "Asclépiades", considérés comme membres d'un " ordre ", et prêtres des sanctuaires (auxquels on attribue certains traits, connus notamment par Galien, de l'histoire des Asclépiades). S'écrivait alors une «pseudo-histoire » de la pratique médicale qui amalgame la tradition héritée de Galien et une certaine représentation de la guérison dans les sanctuaires, et qui place, au nom d'une nouvelle rationalité, son point de départ dans la volonté de condamner les thèses influencées par le messmérisme. Au même moment cependant prenait corps en particulier en Allemagne, une tout autre histoire de la médecine : que ce soit dans sa phase romantique (où on ne dissimule pas une certaine admiration pour les connaissances et les techniques de l'ancienne médecine) ou positiviste (avec, pour le moins, une distance nettement accrue à l'égard des médecins antiques au nom de la «médecine scientifique $)^{14}$, cette autre histoire ignorait bel et bien l'activité des sanctuaires guérisseurs.

Le lien opéré entre les sanctuaires guérisseurs du monde grec et l'histoire de l'ancienne médecine conservait encore de nombreux partisans. Parmi eux, le docteur Vercoutre publie une série d'articles en 1885 et 1886 dans la Revue Archéologique où il se livre à une forme de reconstitution des séjours dans les sanctuaires asclépieiens de l'Antiquité grecque $^{15}$. Tout d'abord, il se concentre sur le rêve et l'action des prêtres. L' ' homme crédule et malade » fait un rêve qui, au moment où il se produit, a "précisément et forcément un rapport étroit avec les idées mêmes qui hantaient son cerveau »; et si l'excitation l'empêchait de dormir, alors la probabilité était forte, dit-il, que le rêve soit remplacé par une hallucination. L'intervention des prêtres est cependant jugée capitale. Pour éviter les « mécomptes » venant des songes des malades eux-mêmes, les prêtres avaient, selon le docteur Vercoutre, imaginé la distinction entre «songes ordinaires ", sans valeur et indignes de toute interprétation, et "songes célestes », les seuls à être envoyés par la divinité et ceux-là mêmes qui se produisent dans un état intermédiaire entre la veille et le sommeil ; or, ayant observé que c'est le matin, à l'aube, que cet état est atteint (ce que Philostrate et Tertullien affirment par ailleurs), les prêtres auraient choisi ce moment pour se présenter en personne au malade, sous le déguisement $\mathrm{du} \mathrm{dieu}^{16}$, et même pour lui adresser la parole. Puis, s'enhardissant, ils n'auraient pas hésité à intervenir à toutes les heures de la nuit, allant jusqu'à se faire escorter par tout un petit groupe de personnages figurant par exemple les « divines sœurs d'Esculape, Hygie et Panacée ». "Ainsi pendant de longs siècles, se joua cette 
petite comédie, et elle fit d'innombrables dupes ${ }^{17}$. " Mais, c'était pour une cause utile puisqu'elle permettait aux prêtres d'intervenir efficacement.

Selon le docteur Vercoutre, en effet, entre le malade crédule et le prêtre imposteur se loge « de la médecine et de la meilleure... ». Il tient la thérapeutique des sanctuaires asclépieiens, «les seuls temples vraiment médicaux », pour rationnelle et scientifique. Chacune des «règles" auxquelles le malade se plie avant le «traitement» est interprétée comme étant destinée à l'instauration de conditions favorables à la guérison : l'arrivée dans un sanctuaire implanté dans un lieu salubre qui contraste avec un pays chaud et fiévreux, les " cérémonies de purification » où les bains assurent en fait la propreté corporelle du malade et constituent une « excellente entrée en matière au début de tout traitement", le sacrifice et les offrandes préliminaires qui « tranquillisent l'âme » et préparent l'amélioration physique tant attendue, la visite du sanctuaire et la contemplation des ex-voto qui engagent " par tous les moyens possibles, le malade à se soumettre avec une confiance aveugle au traitement qui sera institué ", la diète, "prescription éminemment thérapeutique» au commencement d'un traitement... Enfin, au terme de la «supercherie » organisée, les traitements prescrits relèveraient, quant à eux, d'une "pharmacopée sérieuse ": pour ne prendre que les seules inscriptions de lîle Tibérine, à Lucius souffrant d'une pleurésie, le mélange de cendre et de vin qui est prescrit par Esculape ne serait rien d'autre que ce qu' « en langage moderne" on désignerait comme "des frictions alcalino-alcooliques"; à Julianus, souffrant d'une hémoptysie grave, la préparation prescrite (prendre des graines de pin, les mêler à du miel et manger le mélange pendant trois jours) pourrait s'apparenter à l'eau de Brocchieri, remède réputé hémostatique ${ }^{18}$. D'une manière générale, les prêtres seraient également passés maîtres dans la thérapeutique que l'on peut appeler "hygiénique ", Galien affirmant lui-même qu'Asklépios conseillait fréquemment les exercices corporels, tels que la chasse, l'équitation, la gymnastique et l'escrime et, pour ceux qui sont atteints de désordres intellectuels, les spectacles plaisants, la musique ou les chants mélodieux. Voilà qui justifierait l'installation auprès des temples « des gymnases, des établissements balnéaires etc. et dans l'enceinte même du temple d'Épidaure, un théâtre ».

Attribuer ainsi aux " prêtres d'Esculape » une pratique rationnelle nécessitait de traiter de la question de leur « instruction professionnelle ». Dans la lignée des érudits du XIX siècle, le docteur Vercoutre reprend l'idée de l'excellence de l'organisation destinée à assurer l'instruction professionnelle assortie surtout d'une conception du sanctuaire guérisseur comme centre et point de départ d'un savoir transmis ensuite essentiellement par voie d'hérédité. Hippocrate comme Galien se seraient formés à l'ombre des sanctuaires d'Esculape. Toutefois, pour rendre compte d'une documentation qui fait apparaître, dans un bon nombre de remèdes prescrits, des "éléments étranges", il n'hésite pas à doter le prêtre "savant» d'une forme de duplicité qui le conduit à ajouter une auréole de "merveilleux", à délivrer des prescriptions parfois obscures ou allégoriques, ou à intégrer des éléments mystiques : c'est de la confiance du malade et de sa parfaite docilité dont on cherchait ainsi à s'assurer.

15 Le risque était alors réel de verser dans «l'ornière du charlatanisme ", où seraient tombés certains de ces prêtres, «enivrés par leurs succès et surtout enhardis par l'immense crédulité des malades, subissant probablement aussi l'influence quelque peu corruptrice de la domination romaine ${ }^{19} \ldots$ ». Là résidait peut-être la cause du déclin du 
culte d'Esculape, dont la raison principale aurait été cependant la concurrence des autres dieux : "L'Olympe tout entier voulut faire de la médecine » et les prêtres, « tous profondément ignares en médecine... se serviront du même système général, éminemment commode, celui de la prétendue révélation des remèdes par les songes qu'on dit envoyés par la divinitée ${ }^{20}$." Les erreurs de thérapeutique se seraient multipliées, et le public, incapable de faire les distinctions idoines, aurait englobé les uns et les autres dans l'anathème, à l'instar d'un Aristophane raillant le prêtre d'Asklépios dans son Ploutos. Il fallait un Hippocrate, fin connaisseur des prêtres d'Asklépios et averti de ce que leur médecine était vraiment scientifique, pour faire la différence et accuser tous les autres prêtres, ces « copistes serviles et maladroits » des procédés utilisés par les " prêtres d'Esculape ».

16 On le voit, au-delà d'une forme de confusion chronologique (de la «domination romaine " à Hippocrate...), c'est une représentation du malade, du prêtre et de l'histoire de la médecine qui se forme et se développe. Elle domine non seulement bon nombre des écrits $\mathrm{du} \mathrm{xIX}^{\mathrm{e}}$ siècle $^{21}$, mais laisse encore des traces dans la représentation ultérieure que les historiens eux-mêmes ont pu se faire du culte d'Asklépios et du rite de l'incubation ${ }^{22}$. D'une manière récurrente, s'y construit l'image d'un fidèle et d'un malade nécessairement naïf, un individu dont "l'imagination " peut être parfois même grossièrement manipulée. Le prêtre est vu sous la forme d'un démiurge, un "artisan ", détenteur d'une technique de manipulation, initiateur d'un "faire croire» et d'une comédie de rituel, qui cohabite avec un authentique savoir scientifique. Si bien que la confusion s'instaure entre cette figure du prêtre et celle du médecin dont l'érudit du $\mathrm{XIX}^{\mathrm{e}}$ siècle se vit comme l'héritier. Émerge alors un discours destiné à l'expression d'une vérité qui exclut de son champ ce qui relève proprement du fonctionnement $d u$ système religieux et se rapporte à la relation entre l'homme et le dieu. Sous l'apparence d'une scientificité sans faille, un tel discours dissout l'inexplicable et prétend mettre au jour les coulisses d'une mise en scène qu'il veut aussi inscrire dans son histoire de la pratique médicale.

L'édition des stèles d'Épidaure allait-elle être l'occasion d'une rupture entre l'histoire d'une médecine «rationnelle» et l'histoire de l'activité dans les sanctuaires d'Asklépios? D'emblée, à propos de ces inscriptions des $\mathrm{IV}^{\mathrm{e}} / \mathrm{III}^{\mathrm{e}}$ siècle av. J.-C., leur éditeur, P.Cavvadias ${ }^{23}$, souligne qu'il n'est nulle part question de remèdes pharmaceutiques mais seulement de visions et de songes, et que les médecins grecs ne pouvaient en aucun cas tirer profit de tels documents. Il qualifie alors ces guérisons de " miracles » et le sanctuaire n'est pour lui qu'un lieu sacré et non un " hospice », où l'on accourait se placer sous la protection du dieu, se confiant à une "force céleste » et attendant un "miracle ». En s'appuyant sur l'inscription de la seconde stèle (dont le récit de la guérison "miraculeuse " d'une femme souffrant d'un ver intestinal est connu également par l'historien, considéré alors comme contemporain des guerres médiques, Hippys de Rhégion ${ }^{24}$ ), il conclut que les stèles ont été rédigées à partir de témoignages antérieurs (dont l'historien, en l'occurrence, aurait également disposé), par le biais sans doute d'inscriptions placées par les malades guéris sur les ex-voto qu'ils consacraient dans le temple. P. Cavvadias insiste : les stèles d'Épidaure ne sont pas des archives médicales, mais les "diplômes ès-miracles" du sanctuaire. Il concède néanmoins - évoquant l'inscription épidaurienne de Julius Apellas (II siècle ap.J.-C.) dont le récit du traitement et de la guérison est fort long et d'une certaine complexité -, qu'avec le temps, les prêtres d'Asklépios ont dû, pour sauvegarder la réputation du 
sanctuaire, appliquer aux malades les ressources de la médecine proprement dite ${ }^{25}$. Dès lors, il explique l'erreur de ses prédécesseurs qui, parce qu'ils ont confondu ce qui se passait dans les Asklépieia à l'époque grecque et ce qui avait lieu à l'époque romaine, en sont venus à considérer que les prêtres d'Esculape étaient les premiers médecins et qu'Hippocrate, comme l'affirmait Strabon ${ }^{26}$, avait pu profiter du traitement thérapeutique inscrit sur les stèles pour s'instruire. Cette distinction entre deux grandes périodes, l'une au cours de laquelle les guérisons seraient le produit de la puissance de la foi, l'autre, le résultat d'une intervention médicale du personnel, allait en fait poser les bases de la plupart des études et des commentaires ultérieurs portant sur l'activité des sanctuaires guérisseurs.

Enfin, en introduisant la notion de "miracle » pour qualifier les guérisons de ce qu'il considérait comme la première "période" de l'histoire du sanctuaire d'Épidaure, le savant détachait apparemment la question de la guérison au sanctuaire d'une pseudohistoire de la médecine pour l'intégrer à une approche relevant de la sphère religieuse. Mais en réalité, il introduisait une approche des guérisons qu'un certain nombre de savants ne pouvait admettre. Une sorte de scepticisme arrêtait d'emblée ceux qui se refusaient à envisager les guérisons comme contraires aux lois naturelles et comme le produit de la puissance de la foi. Salomon Reinach qualifiait ainsi les récits de "relations puériles", tandis qu'E. Thraemer les considérait comme autant de " légendes pieuses ", à ranger avec les « cures mythologiques » d'Asklépios relatées par Pindare ou d'autres auteurs ${ }^{27}$. Dans l'article "medicus" qu'il écrit pour l'encyclopédie de Daremberg et Saglio ${ }^{28}$, S. Reinach distingue une "médecine sacerdotale» ou «théurgique » d'une "médecine expérimentale ou rationnelle»; la seconde ne doit rien à la première à laquelle, dit-il, elle donna tout ce que celle-ci comportait de rationnel. C'est ce que montre, selon lui, l'inscription de Julius Apellas ${ }^{29}$ où les détails du traitement diététique et psychique traduisent l'apport de la médecine scientifique aux traitements des maladies dans les sanctuaires à une époque tardive. Il condamne sans appel la tradition transmise par Strabon selon laquelle Hippocrate aurait appris de la lecture des inscriptions du sanctuaire de Cos la nature des maladies et de leurs traitements adaptés; il dénonce également la confusion entre les Asclépiades, dont Hippocrate était un représentant à Cos, et les prêtres des sanctuaires guérisseurs. Le seul élément propre à la médecine sacerdotale était, selon S. Reinach, la suggestion, " méthode curative commune à tous les charlatanismes ${ }^{30}$ ".

Dans cette lignée, d'autres chercheurs en sont venus à distinguer soigneusement entre les sanctuaires. D'un côté, Epidaure aurait été le lieu des «miracles mensongers », de l'autre cos et Pergame disposeraient de sanctuaires assimilables à des stations thermales, où la pratique médicale est associée à des interprétations de rêves, à partir desquelles les prêtres déterminent les traitements conçus selon les procédures thérapeutiques rationnelles ${ }^{31}$. Et, s'il peut arriver qu'un miracle se produise, c'est l'effet de la suggestion ou de l'auto-suggestion ${ }^{32}$. Toutefois, désireuses de réduire la part de l'inexplicable, certaines analyses se sont efforcées de montrer que sur l'ensemble de l'histoire du culte d'Asklépios, ce sont bien des méthodes rationnelles qui furent utilisées pour conduire les malades à la guérison. Ainsi, A.P. Aravantinos, dans un ouvrage paru à Leipzig en 1907 et préfacé par M.J. Pagel, professeur d'histoire de la médecine à l'université de Berlin ${ }^{33}$, observe en médecin les dispositions architecturales des sanctuaires, savamment conçues dans l'intérêt des malades, et décrypte le récit des guérisons des inscriptions. Il diagnostique la maladie dont souffrait le fidèle puis s'efforce de montrer que les procédés prêtés au dieu ou à ses ministres sont bien 
l'application de méthodes rationnelles, dans lesquels on reconnait, notamment par le rapprochement avec les textes d'Hippocrate, l'expérience des praticiens du temps.

Une fois les stèles d'Épidaure publiées, la question de la guérison reste finalement structurée par une opposition entre science et religion. Et c'est en ces termes qu'elle reste au centre de bon nombre des études traitant de près ou de loin du rite de l'incubation. Dans leur grande synthèse sur le culte d'Asklépios, E.J. et L. Edelstein ${ }^{34}$ ont séparé en deux chapitres distincts l'étude de ce qu'ils nomment « Temple medicine » et l'étude du culte proprement dit. La première partie traite des guérisons survenues dans le sanctuaire, depuis l'apparition du culte jusqu'à l'époque impériale, avec une attention assez grande portée aux «stèles de guérison » d'Épidaure qui posent, selon les auteurs, le problème des guérisons « miraculeuses ». Le phénomène s'expliquerait pour l'essentiel par la diffusion auprès du public des connaissances médicales et des moyens thérapeutiques utilisés couramment par les médecins. Asklépios y est assimilé à un " patron » des médecins, opérant, traitant et prescrivant comme un médecin, et les cures qu'il prescrit sont la rationalisation de ce que les malades croyaient avoir vu en rêve. Quant aux guérisons instantanées, elles peuvent être l'indice de cas de troubles nerveux ou, pour les cas les plus invraisemblables, le produit d'un miracle du type de celui que, parfois, un médecin appelle de ses vœux quand, devant un cas désespéré, il s'exclame : «Il n'y a qu'un miracle pour le sauver ${ }^{35}$ ! » Le relief ainsi donné à la question des "guérisons " divines débouche sur une étude de leurs implications sociales et politiques : celles-ci constituent comme le complément nécessaire aux guérisons dues aux soins médicaux à une époque où les possibilités offertes par la médecine devaient être limitées.

21 Le culte dans son ensemble, les rites sacrificiels, les processions et les concours organisés en l'honneur du dieu sont définis comme étant la contrepartie nécessaire pour que des guérisons adviennent, une manière pour les hommes d'appeler sur eux le soutien de la divinité. La relation et l'échange entre l'homme et le dieu sont relégués dans une opposition entre les guérisons obtenues d'un côté et un ensemble de rites accomplis collectivement par les hommes de l'autre. L'incubation elle-même n'est pas considérée dans son caractère rituel mais seulement comme un mode d'accès à la guérison. Rêves et visions d'Asklépios sont à comprendre comme relevant de l'accomplissement d'un vœu ou encore le fruit de la mémoire qui fait que s'intègre au rêve la connaissance, puisée dans la vie quotidienne, de traitements prescrits par les médecins. Si L. Edelstein a défendu l'idée qu'il n'y avait pas, dans le monde grec, de solution de continuité entre guérisons « au sanctuaire » et guérisons dues au médecin, il n'en restait pas moins que la dimension religieuse de l'incubation était reléguée au second plan et que les guérisons relevaient pour l'essentiel de la connaissance diffuse d'une science médicale.

Inversement, quelques savants ont insisté sur le fait que le rite de l'incubation et le passage dans un sanctuaire guérisseur relèvent de l'expérience religieuse. De ce point de vue, dans son Histoire de la divination dans l'Antiquité parue au tournant des années 1870-1880, A. Bouché-Leclercq avait marqué quelques points décisifs ${ }^{36}$. En cherchant à différencier l'incubation de l'oniroscopie, il la reconnaissait d'abord comme une forme de mantique, intégrée à la catégorie de la divination «intuitive » - celle que Cicéron aurait qualifiée de «naturelle »: «L'incubation diffère de l'oniroscopie ordinaire en ce qu'elle implique, de la part du consultant, une préméditation et un acte préparatoire ${ }^{37}$. » La dimension rituelle était soulignée, de même que la nécessité, pour 
la pratiquer, de se rendre «dans les temples, où cette coutume créait par là même un oracle». Rattachant l'incubation au culte de quelques dieux et héros, A. BouchéLeclercq s'interrogeait, entre autres, sur sa relation à la guérison dans le chapitre consacré aux «oracles d'Asklépios» et il cherchait à poser la distinction entre Asklépiades et oracles médicaux, même si certains d'entre eux furent, selon lui, écoles d'Asklépiades ou délégations de ces derniers ${ }^{38}$. Mais c'est l'étude de L. Deubner, De incubatione capita quattuor, publiée à Leipzig en 1900 qui pouvait apparaître comme décisive ${ }^{39}$.

Le premier chapitre ("De somniis divinis") s'ouvre sur une étude du vocabulaire du songe qui s'appuie essentiellement sur les distinctions faites par plusieurs auteurs de l'époque impériale (Artémidore, Macrobe); cette démarche conduit l'auteur à faire entrer l'incubation dans la sphère de la divination ou de la mantique. Plus loin, il analyse les modalités de l'apparition de la divinité au cours du rêve (le dieu apparaît sous les traits de sa statue, ou des voix se font entendre au rêveur, ou le rêveur perçoit une lumière...). Dans le second chapitre ("De incubandi ritibus symbolisque"), il distingue l'analyse de certains rites qui entourent le sommeil de l'incubation (interdits alimentaires, sacrifices...) et celle des «symboles » qui constituent l'environnement du rite avec, en particulier, une grande attention accordée aux animaux (le chien, le bélier, le coq...). Malgré le fait qu'il limite l'incubation à une pratique divinatoire, c'est une forme d'approche phénoménologique que fournit L. Deubner, par laquelle le sommeil de l'incubation proprement dit et, incidemment, la relation du fidèle au dieu, deviennent l'objet même de la réflexion.

Tout en reprochant à L. Deubner sa volonté de reconstituer une incubation à « visage unique " et de la réduire à une pratique divinatoire sur l'ensemble de l'Antiquité grecque, Th. Lefort se plaçait dans le même champ d'observation qui est celui du rite ${ }^{40}$. Il distingue d'un côté l'incubation aux $v^{e}$ et $\mathrm{IV}^{\mathrm{e}}$ siècles, époque des thaumata au cours de laquelle ce rite est étranger à l'iatromantique (il ne s'agit pas pour les fidèles d'acquérir une connaissance surnaturelle ou une révélation mais simplement d'obtenir de la puissance divine des thaumata, une guérison directe par exemple), et d'un autre côté l'incubation à l'époque romaine où le rite serait devenu un rite iatromantique, destiné à apporter au fidèle endormi des données qui restent à interpréter pour le diagnostic, ou pour le pronostic, ou encore pour le traitement ${ }^{41}$. C'est cette distinction que reprend peu de temps après, en 1909, O. Weinreich qui, s'interrogeant sur les guérisons miraculeuses dans l'Antiquité, décrit les guérisons par le rêve en distinguant deux types : les guérisons qui se font sans moyen extérieur, par l'action du dieu, et celles qui arrivent parce que le malade obtient du dieu une indication à suivre, la connaissance d'un moyen médical, une action rituelle ou magique à accomplir ${ }^{42}$.

Dans son Incubation or the Cure of Disease in Pagan Temples and Christian Churches, publié en $1906^{43}$, M. Hamilton envisage elle aussi l'incubation comme une pratique divinatoire, " the method by which men sought to entice [some] dreams ${ }^{44}$ ", attachée aux cultes des divinités chthoniennes et des héros investis des pouvoirs que les Anciens auraient attribués à la terre: celui d'envoyer des rêves et d'apporter la guérison. En conséquence, les sanctuaires de ces divinités sont aussi «the centres of medical divination", où se pratique l'incubation dont l'objet est "to meet with the deity in sleep, ask questions, and receive answers ${ }^{45} »$. M. Hamilton s'efforce alors de rassembler les données, littéraires, épigraphiques et archéologiques, sanctuaire par sanctuaire. Pour le sanctuaire d'Épidaure, le commentaire qui suit la traduction des stèles de 
guérison découvertes par P. Cavvadias s'emploie à dégager à la fois la physionomie du rite et celle de la divinité : Asklépios y est décrit comme un pourvoyeur de "miracles ", dont la réputation est si bien établie qu'il peut faire l'objet d'une comédie satirique du type de celle qu'Aristophane a conçue dans son Ploutos. Reprenant à son compte la catégorie du miracle, M. Hamilton concède cependant ne pas savoir jusqu'à quel point les guérisons étaient miraculeuses et s'interroge sur la part éventuelle de l'art de la médecine. À partir de là, elle se rallie au point de vue de P. Cavvadias pour qui l'art médical n'était pas pratiqué pendant les premiers siècles de la prospérité du sanctuaire ${ }^{46}$.

Le deuxième volet de cette enquête sur la pratique de l'incubation aux époques tardohellénistique et romaine cherche, en revanche, à mettre en évidence le rôle de la science médicale et des traitements hygiéniques : le dieu ne sauve plus désormais par son action personnelle, mais il montre les chemins de la guérison; les prêtres jouent dès lors un rôle essentiel dans l'interprétation de rêves qui fournissent les prescriptions idoines. L'analyse du rituel cède bel et bien le pas devant la question des guérisons envisagée selon une double polarité science et religion, celle-là même qui guide également la lecture des inscriptions provenant des sanctuaires de l'île Tibérine à Rome et de Lébèna en Crète. Un curseur s'est, semble-t-il, déplacé entre ces deux pôles pour chacun des récits, au point qu'une des inscriptions de Lébèna, qui concerne la femme guérie d'un ulcère au doigt après que le dieu lui a prescrit un onguent ${ }^{47}$, est désignée comme se référant à une "guérison semi-rationnelle». L'expression est révélatrice : la prescription de l'onguent est banale et attendue en médecine, mais ses propriétés guérisseuses ont dû être décuplées par la croyance de la patiente ${ }^{48}$. Les études qui avaient tenté dans la première moitié $d u x^{e}$ siècle de renouveler l'approche du rite de l'incubation ont donc été à nouveau happées par la question du miracle, mais l'analyse des cas de guérison pouvait cependant être envisagée selon une possible complémentarité entre science et religion.

La découverte puis l'édition, à la fin du XIX ${ }^{\mathrm{e}}$ siècle, des récits de guérison des stèles d'Épidaure n'avait pas ouvert la rupture à laquelle on pouvait s'attendre, ni fait entrer entièrement l'histoire des sanctuaires guérisseurs dans l'histoire des pratiques religieuses. L'analyse des inscriptions d'Épidaure, en substituant immédiatement à la question de la guérison celle du "miracle ", avait réintroduit paradoxalement et du même coup la question de la science. Le concept de "miracle", emprunté au vocabulaire religieux chrétien, était difficilement acceptable pour un certain nombre de savants et les réactions aux premières publications sur les stèles de guérison d'Épidaure allaient de facto conduire à renouer, au moins partiellement, avec les analyses du XIX siècle marquées par une forme d'obsession " cartésienne ». L'approche fondée sur une forme d'opposition, voire d'exclusion réciproque, entre science et religion n'a cessé, au moins jusqu'au milieu du $\mathrm{xx}^{\mathrm{e}}$ siècle, de guider bon nombre d'études, y compris lorsqu'il s'est agi de distinguer des variantes, selon les périodes et selon les sanctuaires, dans les modalités supposées de l'intervention du dieu auprès des fidèles. Certains travaux, cependant, se sont heurtés au caractère réducteur de cette opposition, ce qui a pu ouvrir la voie vers l'idée d'une possible complémentarité des approches. L'histoire de la question ne s'arrête cependant ni avec la somme des Edelstein, ni avec les travaux sur les rites dont L. Deubner avait ouvert la voie ${ }^{49}$. Il a toutefois paru nécessaire et important de faire l'état de cette "première" historiographie tant il est vrai qu'elle a installé durablement des présupposés et des 
choix conceptuels qui ont continué d'informer, au moins partiellement, les recherches ultérieures.

\section{NOTES}

1. S. REINACH, "Chronique d'Orient", Revue Archéologique 4 (1884), p. 76-84 ; les stèles de guérison étaient mentionnées par Pausanias : Pausanias II, 27, 3 ; 36, 1. Pour l'édition de P. Cavvadias, cf. Archaiologike Ephemeris 1883, p. 197-228 (stèle A) ; Archaiologike Ephemeris 1885, p. 1-28 (stèle B) et 85-86 (stèle D) ; Fouilles d'Épidaure, Athènes, 1891, p. 23-32 (stèles A, B et D avec traduction française). Sur les révisions apportées aux premières éditions, cf. L. LIDONNICI, The Epidaurian Miracle Inscriptions. Text, Translation and Commentary, Atlanta, 1995, p. 15.

2. E.J. \& L. EDELSTEIN, Asclepius. A collection and interpretation of the testimonies, I et II, Baltimore, 1945 ; pour la dernière édition, Baltimore, 1998, avec une nouvelle introduction par Gary B. Ferngren.

3. H. МЕІвом, Exercitatio philologico-medica de incubatione in fanis deorum medicinae causa olim facta etc, Helmstadt, 1659. Protestant, fils du médecin Johann Heinrich Meibom (1590-1655), Heinrich Meibom est né en 1638. Il étudia à Helmstadt, à Groningue, et à Leyde avant d'entreprendre divers voyages d'études en Italie, en France et en Angleterre. En 1663, il reçoit à Angers son grade de docteur et en 1664 il prend la chaire de médecine à l'université d'Helmstadt ; à partir de 1678, il devint aussi professeur d'histoire et de poésie dans cette même université où il exerça son activité jusqu'à sa mort en 1700 ; cf. J.-E. DEZEIMERIS, Ch.-P. OLLIVIER, J. RAIGE-DELORME, Dictionnaire historique de la médecine ancienne et moderne, t. III, $2^{\mathrm{e}}$ partie, Paris-Bruxelles, 1837, p. 563-565.

4. A.-M.-J. de CHASTENET, marquis de Puységur, Mémoires pour servir à l'histoire et à l'établissement du magnétisme animal, Paris, 1784 [Toulouse, 1986] ; cf. R. DARNTON, La fin des lumières : le mesmérisme et la Révolution, Paris, 1968, passim.

5. C.A. KOENIG, Dissertatio inauguralis medica de Aristides incubatione, Iéna, 1818 ; on trouve les traces des théories de Messmer dans l'étude plus ancienne de F.A. Wolf's, "Beitrag zur Geschichte des Somnambulismus aus dem Alterthum", Vermischte Schriften und Aufsätze lateinischer und deutscher Sprache, Halle, 1802, p. 382-430 (étude dont la première édtition remonte à 1787) et dans celle de J.F.A. KINDERLING, Der Somnambulismus unserer Zeit mit der Incubation oder dem Tempelschlaf und Weissagungstraum der alten Heiden in Vergleichung gestellt, Dresde, 1788 (Allgemeine deutsche Bibliothek, 87/1, 1789, p. 174-175).

6. A. GAUTHIER, Introduction au magnétisme, examen de son existence depuis les Indiens jusqu'à l'époque actuelle, sa théorie, sa pratique, ses avantages, ses dangers et la nécessité de son concours avec la médecine, Paris, 1840.

7. En particulier chap. VIII et IX.

8. GAUTHIER, Recherches historiques, p. 12.

9. Ibid., p. 20.

10. Ibid., p. 32.

11. Ibid., p. 52.

12. Ibid., p. 51.

13. Ibid., p. 70-71.

14. Cf. par exemple J. HECKER, Geschichte der Heilkunde : nach den Quellen bearbeitet, 2 vol., Berlin, 1822-1829. L'approche positiviste est ouverte avec C. A. WUNDERLICH, Geschichte der Medizin, 
Stuttgart, 1859 ; en France, on le sait, Émile Littré, médecin et philologue, disciple d'Auguste Comte, marque un tournant avec la publication des Euvres complètes d'Hippocrate (édition critique, traduction et commentaire), 10 volumes, entre 1839 et 1861 ; dans le sillage, on trouve Ch. DAREMBERG, Histoire des sciences médicales, comprenant l'anatomie, la physiologie, la médecine, la chirurgie et les doctrines de pathologie générale, 2 vol., 1870.

15. Dr VERCOUTRE, "La médecine sacerdotale dans l'Antiquité grecque", Revue Archéologique 6 (1885), p. 273-292; "La médecine sacerdotale dans l'Antiquité grecque (suite)", Revue Archéologique 7 (1886), p. 22-26 et 106-123.

16. Philostrate, Vie d'Apollonios, I, 8 ; II, 35-36 ; Tertullien, De l'âme, XLVIII. Cette hypothèse est reprise par M. BESNIER, L'île Tibérine dans l'Antiquité, Paris, 1901, p. 225-226.

17. Dr VerCoutre, "La médecine sacerdotale dans l'Antiquité grecque", Revue Archéologique 6 (1885), p. 283-284.

18. VERCOUTRE, "Médecine sacerdotale", p. 286 ; sur ces inscriptions, cf. P. SINEUX, "Pour une relecture des récits de guérison de l'Asklépieion de l'île Tibérine", in Roma Illustrata, Représentations de la ville. Actes du colloque international de Caen (6-8 octobre 2005), Caen, 2008, p. 393-405.

19. Dr VERCOUTRE, "La médecine sacerdotale dans l'Antiquité grecque (suite)", Revue Archéologique 7 (1886), p. 106.

20. VERCOUTRE, "Médecine sacerdotale (suite)", p. 111.

21. Dans la lignée des analyses du Dr Vercoutre et largement appuyé sur elles cf., par exemple, l'opuscule du Dr courToIs-SUfFIT, Les temples d'Esculape. La médecine religieuse dans la Grèce ancienne, Paris, 1891.

22. Cette figure du prêtre-médecin a perduré bien au-delà du XIX ${ }^{\mathrm{e}}$ siècle; cf., par exemple, $\mathrm{P}$. LÉVÊQUE, L'aventure grecque, Paris, 1986 [1éd., 1964], p. 477 : «Les succursales d'Épidaure, notamment cos et Pergame, se couvrent de somptueux édifices qui témoignent de leur richesse. Ces sanctuaires deviennent d'abord de véritables écoles de médecine, d'autant que les miracles se font rares et que maintenant le dieu guérit le plus souvent grâce aux traitements imposés par ses prêtres-médecins. »

23. Supra note 1.

24. Élien, Hist. Anim. IX, 33.

25. Première édition de l'inscription de Julius Apellas (160 ap. J.-C.), P. CAVVADIAS, Archaiologike Ephemeris (1883), p. 227-236, nº 60 ; P. CAVVADIAS, Fouilles d'Épidaure, Athènes, 1891, p. 115 ; "Sur la guérison des malades au hiéron d'Epidaure", in Mélanges Perrot. Recueil de mémoires concernant l'archéologie classique, la littérature et l'histoire anciennes, Paris, 1903, p. 41-43. Pour les nombreuses rééditions, IG IV ${ }^{2}, 1,126$; M. GIRONE, Iamata. Guarigioni miracolose di Asclepio in testi epigrafici, Bari, 1998, p. 58-70.

26. Strabon XIV, 2, 19 (à propos de l'Asklépieion de Cos).

27. E. THRAEMER, s.v. “Asklepios”, P.W., II, 2, 1896, col. 1642-1697, en particulier, col. 1686.

28. S. ReINACH, s.v. "medicus", in Ch. DAREMBERG-E. SAGLio, Dictionnaire des Antiquités grecques et romaines, III, 2, Paris, 1904, p. 1669-1700.

29. Supra note 25.

30. REINACH, "medicus", p. 1671.

31. Cf. les remarques de L. COHN-HAFT, The Public Physicians of Ancient Greece, 1956, p. 63, n. 40.

32. R. HERZOG, Die Wunderheilungen von Epidauros (Philologus, supplément, XXII, 3), Leipzig, 1931, p. 67 et p. 138 sqq.

33. A.P. ARAVANTINos, Asklèpios kai Asklèpieia, Leipzig, 1907.

34. E.J. \& L. EDELSTEIN, Asclepius. A collection and interpretation of the testimonies, I et II, Baltimore, 1945. C'est à Ludwig Edelstein que l'on doit la thèse soutenue dans le second volume. Pour une approche de l'œuvre de L. Edelstein dans son ensemble, cf. O. TEMKIN-C.L. TEMKIN, “Editors' 
Introduction", in O. TEMKIN-C.L. TEMKIN (ed.), Ancient Medicine : selected papers of Ludwig Edelstein, Baltimore, 1967, vii-xiv.

35. EDELSTEIN, Asclepius, p. 173.

36. A. BOUCHÉ-LECLERCQ, Histoire de la divination dans l'Antiquité, Grenoble, 2003 [Paris, 1879-1882 (4 volumes)].

37. BOUCHÉ-LECLERCQ, Divination, p. 221 ; Cicéron, De la divination, I, 72 ; selon la classification binaire adoptée par A. Bouché-Leclercq, la divination « intuitive » s'oppose à la divination « inductive ».

38. BOUCHÉ-LECLERCQ, Divination, p. 733-748.

39. L. DEUBNER, De incubatione capita quattuor, Leipzig, 1900.

40. Th. LEFORT, "Notes sur le culte d'Asclépios. Nature de l'incubation dans le culte", Le Musée Belge 10 (1906), p. 21-37 et p. 101-126.

41. Sur cette définition, notons que Th. Lefort reprenait A. Bouché-Leclercq ; cf. BOUCHÉ-LECLERCQ, Divination, p. 731-732.

42. O. WEINREICH, Antike Heilungswunder. Untersuchungen zum Wunderglauben der Griechen und Römer (RGVV VIII), Giessen, 1909.

43. M. HAMILTON, Incubation or the Cure of Disease in Pagan Temples and Christian Churches, Londres, 1906.

44. Ibid., p. 2.

45. Ibid., p. 3.

46. De ce point de vue, la critique que lui porte J. Gessler de n'avoir pas su opposer deux périodes comme Th. Lefort l'avait fait est injustifiée et curieusement empreinte de relents misogynes : J. GESSLER, "Notes sur l'incubation et ses survivances", Mélanges Th. Lefort, Le Muséon 59 (1946), p. 661-670, et p. 662-663 en particulier. Tout au plus peut-on remarquer que l'introduction générale de l'ouvrage semblait se contenter d'une définition minimale de l'incubation alors que celle-ci est présentée de manière plus complexe au fil de la lecture de la documentation.

47. IC, I, XVII, 19 ; M. GIRONE, Iamata : guarigioni miracolose di Asclepio in testi epigrafici, Bari, 1998, III. 10, p. 108-111.

48. HAMILTON, Incubation, p. 70-71.

49. Pour introduire les tâtonnements de la seconde moitié du $x^{e}$ siècle, nous signalerons ici seulement trois études, de nature, de dimension et de portée différentes : C.A. MEIER, Ancient Incubation and Modern Psychotherapy, Northwestern University Press, 1967, trad. par M. Curtis (Antike Inkubation und moderne Psychotherapie, Studien aus dem C.G. Jung-Institut, vol. 1, Zürich, 1949) a tenté, sans véritable succès, d'adapter la psychanalyse à la question ; A. TAFFIN, "comment on rêvait dans les temples d'Esculape", Bulletin de l'Association Guillaume Budé 1960, p. 325-366, a proposé une mise au point équilibrée, souvent citée, mais en définitive trop peu étayée. Une part du renouveau est sans aucun doute venue de la réédition et des interprétations nouvelles données d'un certain nombre d'inscriptions dont, en 1995, celles des stèles de guérison d'Épidaure : LIDONNICI, Epidaurian Miracle Inscriptions.

\section{RÉSUMÉS}

Jusqu'à la fin du XIX ${ }^{e}$ siècle, une fois éteinte la thèse du "magnétisme " chère aux disciples de Messmer, les différentes approches de l'activité des sanctuaires guérisseurs du monde grec antique et des guérisons qui y survenaient se sont fondées essentiellement sur leur rattachement 
à une histoire de la médecine : il s'agissait alors de dévoiler le jeu de dupes au centre duquel le malade, dévot et naif, s'en remettait à un personnel qui, tout en agissant conformément au savoir médical de l'époque, était aussi maître d'un « faire-croire ». La publication des stèles de guérison d'Épidaure dans les années 1880 n'allait pas amener la rupture à laquelle on aurait pu s'attendre mais temporairement prolonger cette approche structurée selon une double polarité et une opposition entre « science » et " religion ». L'émergence, timide, d'études fondées plutôt sur la complémentarité des approches n'a été rendue possible que lorsque le rite de l'incubation est devenu lui-même un objet défini en tant que démarche ritualisée, intégrée au système religieux des Grecs.

Down to the end of the XIxth century, once the thesis of "magnetism" dear to Messmer's disciples had died out, the various approaches to the activity of the healing shrines of the Antique Greek world and the recoveries that occurred there were essentially founded on connecting them up to the history of medicine: the point then was to expose the fool's game at the centre of which the patient, a naïve votary, gave himself over to a personnel who, while acting in conformity with the medical knowledge of the day, were also past masters in the art of "make-believe". The publication of the Epidauros recovery steles in the eighteen-eighties was not to bring about the rupture that could have been expected but temporarily carry on that structured approach consisting of a twofold polarity and an opposition between "science" and "religion". The unadventurous emergence of studies founded rather on the complementarity of the approaches was made possible only when the rite of incubation became an object defined as a ritualised procedure integrated into the religious system of the Greeks.

\section{INDEX}

Mots-clés : sanctuaire guérisseur, monde grec, Antiquité, histoire de la médecine, rite d'incubation, miracle, guérison

Keywords : healing shrine, Greek world, Antiquity, history of medicine, rite of incubation, miracle, recovery

\section{AUTEUR}

\section{PIERRE SINEUX}

CRAHAM-centre Michel de Boüard (UMR 6273)

Université de Caen Basse-Normandie

pierre.sineux@unicaen.fr 